\title{
An Extension of BSDL for Multimedia Bitstream Syntax Description*
}

\author{
Sylvain Devillers \\ IMEC, Kapeldreef 75, B-3001 Leuven, Belgium \\ Sylvain. Devillers@imec.be
}

\begin{abstract}
In previous works, a generic framework for multimedia content adaptation has been introduced, where XML is used to describe the high-level structure of a bitstream and the resulting description is first transformed by an XSLT style sheet, and then processed to generate an adapted bitstream. In order to provide full interoperability, a new language named Bitstream Syntax Description Language (BSDL) is built on top of W3C XML Schema for the purpose of this generation process. A schema designed in this language and specific to a given coding format allows a generic processor to parse a description and generate the corresponding adapted bitstream. This paper describes an extension of BSDL to provide the new functionality corresponding to the reverse operation, i.e. allowing a generic software module to parse a bitstream conforming to a given coding format described by a schema, and generate the corresponding description. For this, BSDL introduces a number of language mechanisms on top of XML Schema. This paper details these language extensions and reviews the strengths and limits of this approach.
\end{abstract}

\section{Introduction}

The multiplication of new devices gaining access to the Internet makes crucial to be able to propose different versions of the same multimedia content (audio, video or still image) adapted to the client resources in terms of bandwidth, display or computing capabilities. On the other hand, the use of scalable media allows to retrieve different versions of a content from a single file by simple editions and hence saves the burden of generating and handling one file for each required version. However, since each multimedia coding format defines its own data structure, a dedicated software module is required for each offered format in order to properly edit the bitstream and produce the adapted version.

To solve this issue, an original and flexible framework was proposed in [1]. In this method, XML [2] is used to describe the high-level structure of a bitstream; the resulting XML document is called a bitstream description. This description is not meant to replace the original binary format, but acts as an additional layer, similar to metadata. In most cases, it does not describe the bitstream on a bit-per-bit basis, but

* This paper was written while the author was with Philips Digital Systems Laboratories, 51, rue Carnot - B.P. 30192156 Suresnes Cedex, France. 
rather addresses its high-level structure, e.g. how the bitstream is organized in layers or packets of data. Furthermore, the bitstream description is itself scalable, which means it may describe the bitstream at different syntactic layers, e.g. finer or coarser levels of detail, depending on the application. With such a description, it is then possible for a generic software to transform the XML document, for example with an XSLT style sheet [3], and then generate back an adapted bitstream. This method is directly inspired by the web publishing framework that has been developed to dynamically adapt XML content with XSLT style sheets.

It is important to note that while a coding format may be specified as a "flat" structure, i.e. as a sequence of binary symbols, these symbols may be grouped in a hierarchical manner in the description, since the inherent tree structure of an XML document has no impact on the bitstream generation process. Furthermore, the choice of tag names is fully transparent. The design of a bitstream schema for a given coding format is therefore not unique and is fully application dependent.

In order to provide full interoperability, it is then necessary that a processor that is not aware of the specific coding format can nevertheless be used to produce a bitstream from its description. For this, a new language based on W3C XML Schema [4] and called Bitstream Syntax Description Language (BSDL) was introduced in [5]. With this language, it is then possible to design specific bitstream schemas describing the syntax of a particular coding format. These schemas can then be used by a generic processor to parse a description and generate the corresponding bitstream.

A complementary method named gBSDL was also proposed for the case of highly constrained devices where the specific syntax schema is not available. A combined approach is currently being standardized within the MPEG-21 Digital Item Adaptation framework [6] and is described in [7].

This paper takes the BSDL approach a step further by building a new challenging functionality. In order to achieve a fully generic framework, it is necessary for a format-unaware, generic software module to be able to parse a bitstream based on the information provided by its schema and generate its description. In this respect, BSDL comes close to other formal syntax description languages such as ASN.1 [8] or Flavor [9], also known as Syntactical Description Language (SDL) in the context of MPEG-4 [10].

The section 2 presents a brief overview of BSDL, then section 3 details the language features specific to the bitstream parsing process. Lastly, the strengths and limitations of our approach are discussed in section 4.

\section{BSDL Overview}

This section briefly describes how the Bitstream Syntax Description Language (BSDL) introduced above is built on top of XML Schema.

The primary role of a schema is to validate an XML document, i.e. to check that it follows a set of constraints on its structure and datatypes. On top of this, BSDL adds a twofold, new functionality, which is to specify how to generate an adapted bitstream from its description and vice-versa. For this, a number of restrictions and extensions over XML Schema are required.

In the following, we name BSDtoBin Parser the generic software parsing the bitstream syntax description and generating the bitstream, and BintoBSD the software 
performing the reverse operation. BSDtoBin uses a set of XML Schema components for which semantics may be assigned in the context of the bitstream generation. Some components should therefore be ignored because they have no meaning in this context, while other constructs are excluded.

Similarly, BintoBSD uses an additional set of extensions and restrictions. In order to remain compatible with XML Schema, these extensions are introduced as application-specific annotations, namely by using the xsd:appinfo schema component and adding attributes with non-schema namespace. In order to distinguish both levels of extensions and restrictions, we name BSDL-1 the set of language features corresponding to the bitstream generation process, and BSDL-2 the set required for the description generation, where BSDL-1 is a subset of BSDL-2. The sets of extensions are declared in two schemas respectively named BSDL-1.xsd and BSDL2.xsd, the latter importing the previous one. The BSDL-1 specification was described in [5] and will not be repeated here. On the other hand, BSDL-2 has not been published yet and is the subject of this paper. The following section details the restrictions and extensions of BSDL-2 in terms of structural and datatypes aspects, as well as their semantics.

In this paper, the "xsd:" prefix is used as a convention to refer to the XML Schema namespace, while "bsdl:" refers to the BSDL extensions.

\section{BSDL-2: Bitstream Parsing}

\subsection{Structural Aspects of BSDL-2}

A schema or a DTD define a set of constraints on XML documents. In XML Schema, most constraints are static, i.e. they are fully specified by the schema. For example, the minimum and maximum number of occurrences of a particle (xsd:minOccurs and xsd:maxOccurs) can be specified as a constant only and it is not possible to define a number of occurrences depending on the value of some other elements or attributes of the instance. On the other hand, this feature is required for parsing a bitstream since the length of a given field or its number of occurrences may be specified by a parameter found upstream.

BSDL-2 thus needs to introduce new dynamic constraints on top of XML Schema, in particular to specify the number of occurrences of a particle or its conditional occurrence. While BSDL-1 declares types and attributes that carry specific semantics in the context of bitstream generation, but are still validated by XML Schema, these constraints are actually new language extensions. In order to remain fully compatible with XML Schema, these new constructs are added as annotations, namely as attributes with non-schema namespace. The three attributes introduced below are used to characterize a particle as an additional property.

Firstly, BSDL-2 introduces a new attribute named bsdl:nOccurs to specify a variable number of occurrences. It is declared in the BSDL namespace and is used to characterize a particle in the same way as xsd:minOccurs and xsd:maxOccurs. It contains an XPath [11] expression indicating the relevant value as explained below. While parsing a bitstream, BintoBSD progressively instantiates the output description in the form of a DOM tree [12]. At a given stage in the bitstream parsing process, any parameter found upstream has thus already been instantiated as an element node of 
the DOM tree. XPath 1.0 expressions are then used to locate this element relatively to the current element or with an absolute addressing in the DOM tree.

Similarly to bsdl:nOccurs, BSDL-2 introduces an attribute named bsdl:if to specify the conditional occurrence of a particle. It contains an XPath expression that should be evaluated as a boolean value.

Note that since bsdl:nOccurs is ignored by XML Schema, the allowed range declared by xsd:minOccurs and xsd:maxOccurs should cope with the expected value. Similarly, if a bsdl:if is specified, then xsd:minOccurs should be set to zero in case the condition is evaluated as false.

Unlike previous attributes that specify constraints depending on data found upstream, a third attribute named bsdl:ifNext specifies a constraint on data found immediately downstream. It contains an hexadecimal string that should be compared to the next bytes in the stream. The particle is parsed if the sequences of bytes are identical. The syntax of bsdl:ifNext also allows an array of two hexadecimal strings separated by a dash character to specify a range of allowed values. This allows to test a number of bits other than multiple of eight. For example, the element <xsd:element name="elt1" bsdl:ifNext="08-0F"/> is to be parsed if the next four bits in the bitstream are set to zero and the fifth to one.

\subsection{Datatypes Aspects of BSDL-2}

BSDL-2 uses the same datatype model than BSDL-1, but with a set of additional constraints, in particular on types relating to sequence of bytes.

While for some XML Schema built-in datatypes such as xsd:short or xsd:int, the encoding length is known from the schema (here, respectively 2 and 4 bytes), the length of xsd:hexBinary, xsd:base64 and bsdl:byteRange can only be determined from their lexical representation in the instance. It is therefore necessary to constrain their length in the schema so that BintoBSD knows how many bytes should be read. The xsd:length facet is used to constrain the first two types for this purpose. However, it cannot be used for bsdl:byteRange since this type is considered by XML Schema as an array of integers. The xsd:length facet then constrains the number of items and not the segment length.

Furtermore, a new mechanism is required to constrain the length when it is not constant. BSDL-2 introduces a new facet named bsdl:length and declared in the BSDL namespace, which allows to distinguish it from the XML Schema facet. Since XML Schema does not currently allow a user to add his/her own facets, it is added to the xsd:restriction component via the annotation mechanism, i.e. the xsd:annotation/xsd:appinfo combination, and processed by BintoBSD as a regular facet. It contains an XPath expression that should be evaluated as an integer against the instantiated DOM tree, and gives the length in bytes of the data segment.

The Document 1 gives a snippet of a Bitstream Syntax Schema for the JPEG2000 coding format showing the use of a variable value defining the length of the jp2:MarkerData element content.

Document 1. Example of JPEG2000 Bitstream Syntax Schema

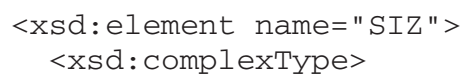




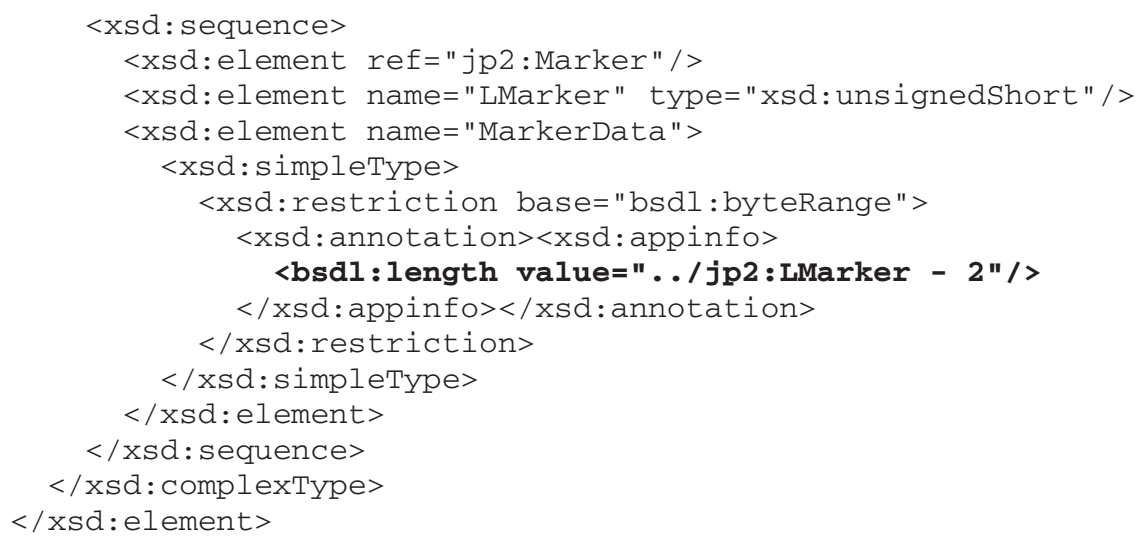

Lastly, several coding formats use start codes to delimit packets of data, which allows to efficiently locate different packets without having to decode their inner structure. In this case, the packet of data may be read as an xsd:hexBinary, xsd:base64 or bsdl:byteRange following the application needs, but since the length is unknown, a mechanism is required to indicate that the bitstream should be read until a given start code is found. For this purpose, we introduce a new BSDL facet named bsdl:startCode which contains the given sequence of bytes written in hexadecimal format. The BintoBSD parser thus reads the bitstream until the given sequence of bytes is found.

\section{Discussion}

We have introduced in this paper a language for describing the syntax of a multimedia bitstream. Among the similar languages proposed in the literature, Flavor [9] is probably the most accomplished attempt. This section discusses the strengths and limits of our approach, in particular in comparison to Flavor.

\subsection{BSDL and XML Schema}

The main characteristic and challenge of our work is that we chose not to develop "yet another language", but rather to build it on top of an existing one. Furthermore, we chose to base it on a declarative language, the function of which is to define constraints on XML documents.

We are conscious that, by doing so, we are using XML Schema for a purpose which is not its initial object. However, several of the introduced features define dynamic constraints on XML documents and are thus still relevant to XML Schema. The bsdl:if attribute states a condition on the occurrence of a particle and bsdl:nOccurs specifies a variable number of occurrences. Lastly, the bsdl:length facet provides the same functionality than xsd:length but with a variable value. In our application, XML Schema and BSDL extensions are first used to validate the description. Then, in a second step, we assign specific semantics to the schema 
components in the form of a processing model for the description and bitstream generation process, not detailed here. Some other constructs such as the bsdl:ifNext attribute or the bsdl:startCode facet are specific to the bitstream parsing process and irrelevant to XML Schema. However, in order to simplify the syntax, we chose to define the two different types of extensions in a single specification and namespace.

\subsection{Use of BSDL for Bitstream Parsing}

It should be noted that BSDL does not and cannot have the ambition to parse any bitstream at any level of detail. Most coding formats have been specified without the use of a formal language (except notably for MPEG-4 Systems written in Flavor/SDL) and do not follow any constraints. In particular, the main part of a bitstream - qualified hereafter as the "payload" - is usually the output of an encoding process such as entropy coding, wavelet or Discrete Cosine Transform. Such sequence of bits can only be decoded via an algorithm implemented in a programming language. It is obviously not the ambition of BSDL or Flavor to natively decode such data.

In order to handle specific encoding schemes, it is possible to extend the BSDL parser. For this, the user-specific datatype should be defined in a schema with an attribute (not described here) indicating that it is hard-coded. The type definition in the schema is used for validating the lexical representation in the description, but not by the BintoBSD parser. The encoding and decoding methods are then implemented following a provided interface and appended to the system classpath (for a Java implementation). While reading the schema, the BintoBSD parser then dynamically instantiates the corresponding class and runs the encoding or decoding method.

On the other hand, we believe that using a formal language to specify a bitstream syntax helps checking its consistency and separating the parsing and decoding processes. While designing a schema for the MPEG-4 Video Object Layer (VOL) format [13 and testing it, we found some inconsistency in the spelling of the mnemonics and some ambiguity on default values of some parameters. Such imprecision is prone to incorrect interpretations and discrepancies in software implementations. The syntax description of MPEG-4 visual part (without the semantics) represents around seventy pages of tables. It is thus a very difficult task, if not impossible, to exclude any error from the text. Writing directly the syntax in a formal language would allow to test and validate its consistency, and track the modifications changes during the specification process.

Furthermore, specifying a bitstream syntax usually consists in two different tasks, firstly in formatting the output of an encoding method as seen above, and secondly in organizing the parameters and packets of data as a stream of symbols. Even though the compactness of the global result is a strong requirement, it is vital to be able to efficiently parse the bitstream to access the different segments of data. Typically, for parallelizing some tasks, the parsing process should be achievable at low cost in order to distribute the data segments to be decoded to dedicated processors. For these reasons, using a formal language implicitly sets some constraints on the bitstream syntax and hence benefits the complexity of the parsing process.

Another main feature of our approach is that it does not only provide a grammar for parsing the bitstream, but also a persistent representation of the bitstream content in the form of an XML document - the description. In comparison, the principle of 
Flavor is to generate $\mathrm{C}++$ or Java code from the bitstream syntax description, which can be interfaced with the application. In this case, the memory representation of the bitstream content is a hierarchy of $\mathrm{C}++/$ Java objects and is thus language dependent. More recently, an extension named XFlavor [14] has been developed to provide an XML representation of the data. On the other hand, BSDL provides both a grammar to parse the bitstream - the schema - and a persistent representation of the data - the description. Having the data in XML format gives access to the extensive family of XML-based languages and tools (XSLT, XPath...). Furthermore, before generating the XML document, the BSDL parser first instantiates a DOM tree, which is a standard, language-independent memory representation of XML data and can thus be easily interfaced with the application.

Lastly, a limit of our approach is the inherent verbosity of XML and XML Schema. Several solutions have been proposed to solve this issue. In particular, MPEG-7 BiM [15] is a very efficient method for binarizing an XML document by using its schema. Since BSDL remains compatible with XML Schema, BiM can be used to binarize the bitstream description, which is particularly useful when descriptions grow large, notably for video. As for XML Schema, a number of commercial products now provide graphical interfaces to design schemas in a user-friendly way. Lastly, it should be reminded that BSDL was primarily introduced for the problem of content adaptation, and thus is meant to describe bitstreams mainly at a high syntactical level, which should limit the verbosity of the descriptions.

\section{Conclusion}

In this paper, we extend the scope of the Bitstream Syntax Description Language (BSDL), which was primarily introduced for the problem of content adaptation. By adding new language mechanisms and defining specific semantics in the context of bitstream parsing, it is possible for a generic processor to use the schema to parse a bitstream. With this respect, BSDL comes close to other description languages such as Flavor. It is interesting to note that both languages tend to the same functionality while BSDL is based on XML Schema and Flavor, though presented as a declarative language, on a $\mathrm{C}++$ like syntax. By using XML Schema beyond its original purpose, we also demonstrate the expressive power of this language. Lastly, we introduce new dynamic constraints on XML documents.

Tests have been successfully performed on several multimedia coding formats, including JPEG2000 images, MPEG-4 Video Elementary Streams and MPEG-4 Visual Texture Coding. Other ongoing tests include MPEG-4 Audio AAC and JPEG.

Although a generic software cannot pretend to be as efficient as an ad-hoc, specific software, the BSDL method significantly facilitates the process of accessing and reading structured, binary data by providing a declarative approach to bitstream parsing.

Acknowledgement. Part of this work was funded by the OZONE research project (IST-2000-30026). 


\section{References}

1. Amielh M. and Devillers S.: Multimedia Content Adaptation with XML, $8^{\text {th }}$ International Conference on Multimedia Modeling MMM'2001 (Amsterdam, The Netherlands, November 5-7, 2001), Paper Proceedings pp. 127-145.

2. XML Extensible Markup Language 1.0 (Second Edition), W3C Recommendation, October 6, 2000.

3. XSL Transformations Version 1.0, W3C Recommendation, November 16, 1999.

4. XML Schema Part 0: Primer, Part 1: Structures and Part 2: Datatypes, W3C Recommendation, May 2, 2001.

5. Amielh M. and Devillers S.: Bitstream Syntax Description Language: Application of XML Schema to Multimedia Content Adaptation, $11^{\text {th }}$ International World Wide Web Conference, WWW2002 (Honolulu, May 6-11, 2002), Alternate Paper Tracks.

6. ISO/IEC JTC 1/SC 29/WG 11/N5353 MPEG-21 Digital Item Adaptation CD, December 2002, Awaji Island, Japan.

7. Panis G., Hutter A., Heuer J., Hellwagner H., Kosch H., Timmerer C., Devillers S. and Amielh M.: Bitstream Syntax Description: A Tool for Multimedia Resource Adaptation within MPEG-21, Accepted to Signal Processing: Image Communication, special issue on Multimedia Adaptation.

8. ASN.1 (ISO Standards 8824 and 8825).

9. Eleftheriadis A.: Flavor: A Language for Media Representation, ACM Multimedia '97 Conference, (Seattle, WA, November 1997), Proceedings pp. 1-9.

10. ISO/IEC 14496-1:2001 Information Technology - Coding of Audio-Visual Objects - Part 1: Systems.

11. XML Path Language, W3C Recommendation, 16 November 1999.

12. Document Object Model (DOM) Level 2 Core Specification, Version 1.0, W3C Recommendation 13 November, 2000.

13. ISO/IEC 14496-2:2001 Information Technology - Coding of Audio-Visual Objects - Part 2: Visual.

14. Hong D. and Eleftheriadis A.: XFlavor: Bridging Bits and Objects in Media Representation, IEEE Int'l Conf. on Multimedia and Expo ICME (Lausanne, Switzerland, August 2002).

15. ISO/IEC 15938-1:2002 MPEG-7: Multimedia content description interface - Part 1 : Systems. See also http://www.expway.tv for BiM. 